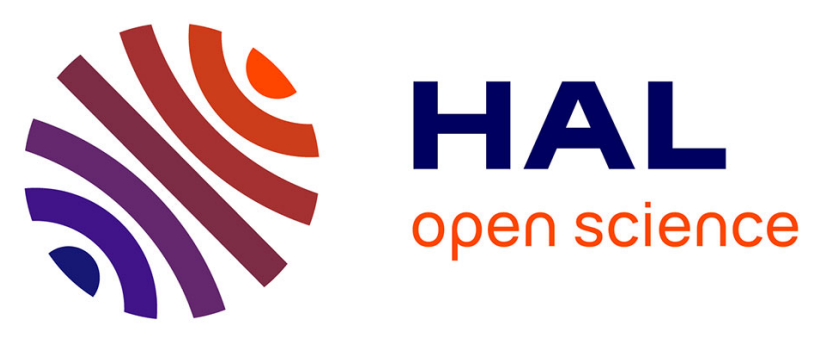

\title{
Medoid-based MLP: an application to wood sawing simulator metamodeling
}

\author{
Sylvain Chabanet, Philippe Thomas, Hind Bril El-Haouzi
}

\section{To cite this version:}

Sylvain Chabanet, Philippe Thomas, Hind Bril El-Haouzi. Medoid-based MLP: an application to wood sawing simulator metamodeling. 13th International Conference on Neural Computation Theory and Applications, NCTA 2021, Oct 2021, Online streaming, Portugal. hal-03404548

\author{
HAL Id: hal-03404548 \\ https://hal.science/hal-03404548
}

Submitted on 26 Oct 2021

HAL is a multi-disciplinary open access archive for the deposit and dissemination of scientific research documents, whether they are published or not. The documents may come from teaching and research institutions in France or abroad, or from public or private research centers.
L'archive ouverte pluridisciplinaire HAL, est destinée au dépôt et à la diffusion de documents scientifiques de niveau recherche, publiés ou non, émanant des établissements d'enseignement et de recherche français ou étrangers, des laboratoires publics ou privés.

\section{(ㅇ)(1) $\$$}

Distributed under a Creative Commons Attribution - NonCommercial - NoDerivatives $\mid 4.0$ 


\title{
Medoid-based MLP: an application to wood sawing simulator metamodeling
}

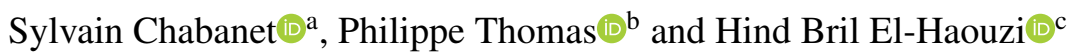 \\ Université de Lorraine, CNRS, CRAN, F-88000 Epinal, France \\ \{sylvain.chabanet, hind.el-haouzi, philippe.thomas\}@univ-lorraine.fr
}

Keywords: $\quad$ MLP, regressor ensemble, Sawmill simulation, ICP dissimilarity, Medoid

Abstract: Predicting the set of lumbers which would be obtained from sawing a log at a specific sawmill is a difficult problem, which complicates short and mid term decision making in this industry. While sawmill simulators able to simulate the sawing of a log from a 3D scan of its outer shape exist, they can be extremely computationally intensive. Several alternative approaches based on machine learning algorithms and different set of features were explored in previous works. This paper proposes the use of one hidden layer perceptrons, and a vector of features build from dissimilarities from the scans to a set of selected wood logs, chosen as the class medoids. Several architectures are tested and compared to validate the pertinence of the proposed set of medoid-based features. The lowest mean squared error was obtained for MISO neural networks with a sigmoid output activation function, to constrain the output value ranges.

\section{INTRODUCTION}

As is reflected by the scientific literature, artificial intelligence tools, including neural networks, could advantageously intervene at different levels of the forest-wood industry. A popular application seems, for example, to be the processing of remote sensing data. For example, (Del Frate and Solimini, 2004) assesses the interest of Multi Layers Perceptron (MLP) models to evaluate forest biomass from airborne laser data. Recently, (Zhang et al., 2020) proposed a lightweight convolutional neural network (CNN) architecture to classify tree species using airborne multi-spectral imagery. Similarly, (Sylvain et al., 2019) uses an ensemble of deep CNN to map dead trees from aerial photographs. An advantage of using remote sensing technologies and artificial intelligence algorithms in these cases is that they limit the need for time consuming and labor intensive ground studies in areas sometime difficult to access on foot. As example of other applications of neural networks to the forest wood industry, (Thomas and Thomas, 2011) uses a MLP to reduce a sawmill workshop simulation model, focusing on bottlenecks. Additionally, (Wenshu et al., 2015) studies the use of Artificial

\footnotetext{
a (iD https://orcid.org/0000-0002-3706-293X

b (iD) https://orcid.org/0000-0001-9426-3570

c (iD) https://orcid.org/0000-0003-4746-5342
}

Neural Networks to classify wood defects on boards. Their final objective is to improve sawmills lumber recovery.

A particularity of the sawing process is that the set of lumbers which can be obtained by processing a specific $\log$ at a sawmill with a given configuration, called in this study a basket of products, can be difficult to predict in advance. This is due, in particular, to the heterogeneity of the raw material. Each $\log$ has a specific shape, quality, and defects, all of which influence the final set of products. In addition, the sawing process itself is divergent with co-production. This means that several lumbers, and other co-products, are simultaneously sawed from the same log. Lumbers sawed from a same log, in particular, may have various dimensions and grades. This introduces uncertainty in the production process and complicates decision making. For example, as explained in (Wery et al., 2018), the acceptation by a sawmill of an order with unusual products would require to change the sawmill configuration. This would impact the whole lumber mix produced, in a way which is difficult to predict. Other studies, like (Morneau-Pereira et al., 2014) propose a Mixed-Integer Programming (MIP) model to optimize the allocation of wood logs from cut blocks to sawmills. Such a MIP model requires, however, at least an estimation of what can be obtained from the set of logs at each sawmill. Both of these authors use simulation tools to generate the data 


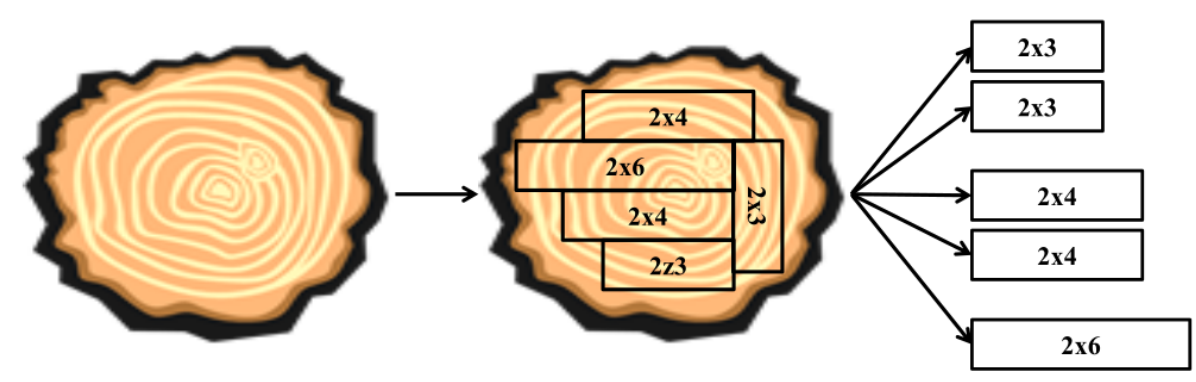

Figure 1: Sawing is a divergent process with co-production. Several lumbers with various dimensions are obtained from the sawing of a single log. Here, two 2"x3" lumbers, two 2"x4" lumbers and one 2"x6" lumber would be sawed from the log.

needed for their respective models.

The forest industry has, indeed, several sawing simulators at their disposal, which are able to digitally break a specific log into a set of lumbers given a 3D scan of the log surface. Examples of such simulators include Optitek (Goulet, 2006), SAWSIM ${ }^{1}$ or Autosaw (Todoroki et al., 1990). These simulators are extremely flexible and effective. A potential issue is, however, that depending on the log considered and simulation settings they can be extremely computationally intensive. This is particularly a problem when multiple simulations have to be run with different logs and sawmills configurations. (Wery et al., 2018) proposes to couple simulation and optimization methods to reduce the number of simulations needed to take a decision. Similarly, (Morneau-Pereira et al., 2014) only runs sawing simulations for a subset of logs at each sawmills, and still report that while solving the MIP took only a few seconds, the simulations required to generate the data needed took several hours.

This paper focuses on another approach centered around the usage of Machine Learning (ML) technologies to build metamodels of these simulators. These metamodels are used to approximate the simulators output, and the prediction process is, usually, fast. Data generated by such metamodels have been used to solve a tactical planning problem in (Morin et al., 2020), shoving an impressive increase of the maximized objective function, when compared with simpler models based only on averaged historical data. Several approaches have been proposed to build these simulators metamodels. In particular, (Selma et al., 2018) and (Chabanet et al., 2021) propose to use pairwise scan dissimilarities and a $\mathrm{k}$ nearest neighbors algorithm. The main contributions of this paper are twofold. First, it further assesses the discriminative power of this dissimilarity by using it to train MLP and MLP ensembles. Second, it com-

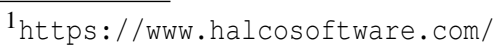
software-1-sawsim, Last accessed on June, 2021
}

pares several MLP and MLP ensembles architectures on this particular problem. The remainder of this paper is structured as follows. Section 2 overviews previous works on sawing simulator metamodelling, and presents the dissimilarity-based representation framework considered. Section 3 describes in details the learning problem considered, as well as the dataset and evaluation scores used in this study. Section 4 presents the different MLP compared in this study, and the way they are, in a second time, aggregated to build predictors ensembles. Results are given in section 5. Section 6 concludes this paper.

\section{Related works}

This section presents related works, both on the problem of sawing simulator metamodeling, and on the problem of learning from dissimilarity data.

\subsection{Sawing simulator metamodelling}

To use ML algorithm for sawing simulator metamodels was first proposed by (Morin et al., 2015). Their proposed metamodels take as input a vector of features describing the log, like its length or diameter, and are trained to approximate the result of a simulator. In particular, their objective is to predict the basket of products which would be obtained by sawing the $\log$ at a given sawmill. However, these ML metamodels only take into consideration a short vector of knowledge-based descriptors while modern simulators are able to use a full 3D scan of the log profile, that is, a 3D point cloud containing thousands of points sampled on the log surface. This leads to a loss of potentially important information which cannot be learned by the metamodels.

Considering that fact, (Selma et al., 2018) proposes the use of a $k$ Nearest Neighbors classifier based on a dissimilarity between the whole scans. This dissimilarity is based on the iterative closest point (ICP) 
algorithm (Besl and McKay, 1992). An inconvenient of this approach is, however, the still important computational cost associated with the computation of multiple pairwise ICP dissimilarities. (Chabanet et al., 2021) later improved upon this method by proposing a set of rules to reduce the number of dissimilarity computation necessary to yield a prediction.

Lastly, (Martineau et al., 2021) proposes the use of specific representations of the point clouds and specialized architectures in combination with knowhow features to train Neural Networks. In particular, they use pointnet (Qi et al., 2017) to learn from a point cloud representation, and $\mathrm{CNN}$ to learn from an image-like representation. They do not consider, however, the use of the previously proposed ICP dissimilarity as input to their networks.

\subsection{Dissimilarity learning}

The use of expert designed dissimilarities rather than expert designed features for pattern recognition has been studied by several authors. A pairwise dissimilarity is defined as a function $d: \mathbf{B} \times \mathbf{B} \mapsto \mathbf{R}$, with $\mathbf{B}$ the space of input data. Intuitively, such a dissimilarity measures how different two objects are, but do not necessarily respects metrics properties such a symmetry or triangle inequality. Two major frameworks developed to use dissimilarity-based information are, on the one hand the embedding of the pairwise dissimilarity matrix $M$ in a pseudo euclidean space, or Krein space, and, on the other hand, the use of the dissimilarity space (Duin and Pekalska, 2009). An interesting review of these methods and others can be found in (Schleif and Tino, 2015).

This paper focuses on the dissimilarity space embedding framework, as it allows to directly use offthe-shelf ML algorithms, including MLP. In this setting, an object $y \in \mathbf{B}$ is represented by a vector $\left(d\left(y, \tilde{x}_{1}\right), \ldots d\left(y, \tilde{x}_{n}\right)\right)$, where $\left\{\tilde{x}_{1}, \ldots, \tilde{x}_{1}\right\}$ is a collection of objects from $\mathbf{B}$, for example a subset of the training dataset.

These objects, called landmarks, may be selected at random or following heuristics and systematic methodologies (Pekalska et al., 2006). In particular, (Cazzanti, 2009) proposes to use class medoids $\left(\mu_{1}, \ldots, \mu_{C}\right)$ as landmarks. A class medoids is an element of a class which minimize the average dissimilarity with all other members of the same class:

$$
\mu_{i}=\underset{y \text { in class } i}{\arg \min } \frac{1}{n_{i}} \sum_{x \text { in class } i} d(y, x), \forall i \in \llbracket 1 . . C \rrbracket,
$$

with $d$ the dissimilarity function, $n_{i}$ the number of points in class $i$ and $C$ the number of classes.

\section{MATERIALS AND METHODS}

This section describes in detail the learning problem considered in this study, as well as the industrial dataset used to train, evaluate and compare the models. Furthermore, the evaluation scores used in this paper are also presented.

\subsection{LEARNING PROBLEM}

The objective of the MLP models studied in this paper is to predict the basket of products which would be obtained by processing a log at a given sawmill, using dissimilarities between 3D scans of the logs surface as inputs. These scans are unordered point clouds containing an arbitrary number of points and, therefore, cannot be used directly by traditional MLPs, but would require specialized architectures or data processing, as was, for example, done in (Martineau et al., 2021). This motivates the use of a pairwise dissimilarity-based learning method.

The outputs of these predictors, the baskets of products, were similarly modeled as vectors. More precisely, considering a set of $p$ individual products present in the dataset, numbered from 1 to $p$, the basket of products is represented as a $p$ dimensional vector. The $i^{t h}$ element of this vector represents the number of lumbers of type $i$ obtained from digitally sawing the $\log$ with a simulator. This output is therefore multidimensional. Furthermore, it can be referred to as structured, as the different dimensions of the vector cannot be considered independent from one another.

The problem of predicting the basket of products of a log given a set of features can be considered either as a classification problem, or as a regression problem. In the classification problem, each type of basket presents in the training dataset is considered as a distinct class, numbered from 1 to $C$, and the objective of the classifier is to assign one of these class labels to new logs. In the regression case, the model directly output a p-dimensional vector, as a prediction of the basket of products.

This study focuses on the regression case. Scans were embedded in a dissimilarity space using the ICP dissimilarity, and landmarks were chosen as the medoids of frequents classes. Classes are, here, defined as the sets of lumbers sharing an exact same basket of products.

\subsection{DESCRIPTION OF THE DATASET}

The dataset used in this study is a proprietary dataset originating from the Canadian forest industry. It is 
composed of 1207 scans of real logs. An example of such a $\log$ scan is presented figure 2 . Each scan can be seen as a table of dimension $N_{p} \times 3$, with $N_{p}$ the number of points, which changes from one scan to another, with an average of 12000 points per scan. Each of the 3 points coordinates corresponds to one column in the scan table.

Additionally, points, are roughly organized as ellipsoids which, together, span the log surface. Originally, the scans contained sections with missing ellipsoids, which led to poor performances of the ICP algorithm when computing logs dissimilarities. This was corrected by filling these empty sections with repetitions of the ellipsoid which immediately precedes them.

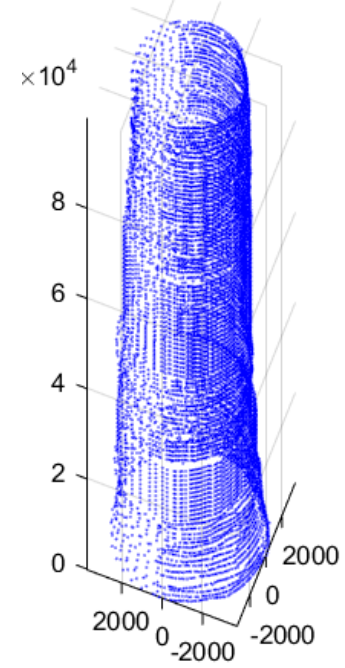

Figure 2: Example of a 3D full profile scan of a $\log$

The basket of products associated with each $\log$ was generated by the sawing simulator Optitek. The dataset contains 19 different types of individual products, characterized by their length, width and thickness. Therefore, the structured output vector one has to predict is of dimension 19. It might be noticed that no basket contains more than 5 different types of products and that, therefore, these vectors are sparse.

For training, selection and evaluation purpose, the dataset was randomly divided into three parts, that is, a training set containing $600 \operatorname{logs}$, a validation set containing $400 \operatorname{logs}$, and an evaluation set containing the remaining 207 logs. The training set contains only 62 different classes, which should lead to 62 medoids used to build features. However, most of these classes appear only once in the dataset. Therefore, to reduce the dimension of the input vector, composed of dissimilarities toward these class medoids, a medoid is considered to induce a feature only if it corresponds to a class with more than two elements in the training set. This reduces the dimension of the input vector to 21.

A drawback of using the ICP dissimilarity in our case is, however, its behavior when comparing logs of different lengths. When comparing a long log with a short one, the value of the dissimilarity explodes compared with the normal range of values when using short logs only. This creates unwanted but extremely high correlations between the 8 features built from dissimilarities to shorter logs. To solve this issue, these features were clipped to 3000 , as is shown in figure 3.

\subsection{EVALUATION SCORES}

As per usual, evaluation scores have to be defined to evaluate and compare the different models. Several set of scores were introduced specifically for the problem of evaluating sawing metamodels. It was, indeed, observed that in a classification setting, classic scores like the 0-1 score don't take into consideration the fact that the cost of missclassification might vary among different real/predicted pairs, while in a regression setting, the squared error is difficult to interpret. Therefore, the prediction score, $s^{\text {pre }}$, production score, $s^{\text {pro }}$ and prediction-production score, $s^{\text {pre } \times \text { pro }}$ were defined by (Morin et al., 2015). Similarly, adaptation of the classic precision, recall and $F_{1}$ scores were introduced in (Martineau et al., 2021).

First, considering that the vectors representing the baskets of products are sparse, to let all the $(0,0)$ real/predicted pairs in these vectors might biased $s^{\text {pre }}$, $s^{\text {pro }}$, and $s^{\text {pre } \times \text { pro }}$ optimistically, all such pairs are removed. Consider $\tilde{p}$ the length of the filtered vectors. The prediction score is then defined as:

$$
s^{p r e}(y, \hat{y})=\frac{1}{\tilde{p}} \sum_{i=1}^{\tilde{p}} \min \left(1, \frac{\hat{y}_{i}}{\max \left(\varepsilon, y_{i}\right)}\right),
$$

with $\varepsilon$ a small value to avoid dividing by zero. $y_{i}$ and $\hat{y}_{i}$ are the $i^{t h}$ components of the real and predicted vectors respectively. This score can be seen as the per product average proportion of the real basket which is effectively predicted.

Similarly, the production score is defined as:

$$
s^{p r o}(y, \hat{y})=\frac{1}{\tilde{p}} \sum_{i=1}^{\tilde{p}} \min \left(1, \frac{y_{i}}{\max \left(\varepsilon, \hat{y}_{i}\right)}\right),
$$

and can be seen as the per product average proportion of the prediction which is effectively produced. 
(a)

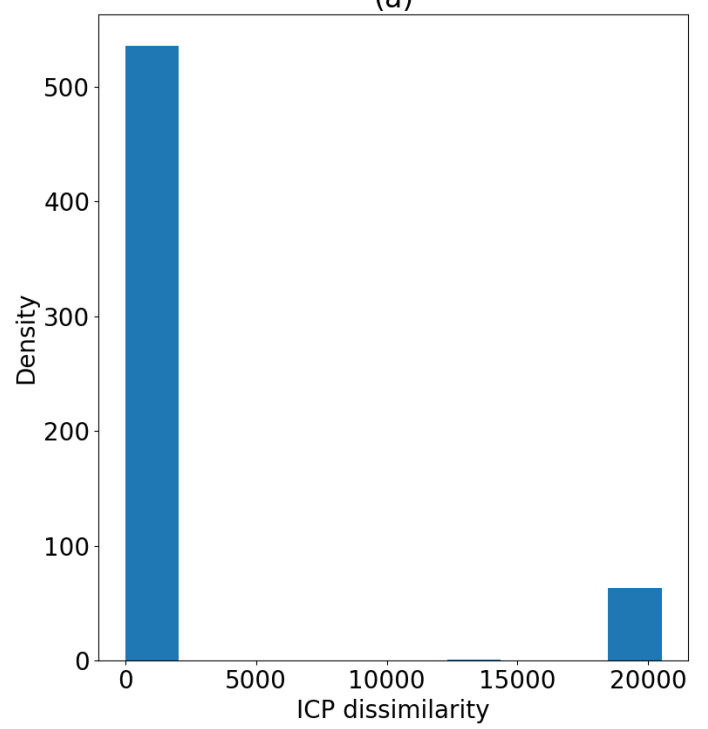

(b)

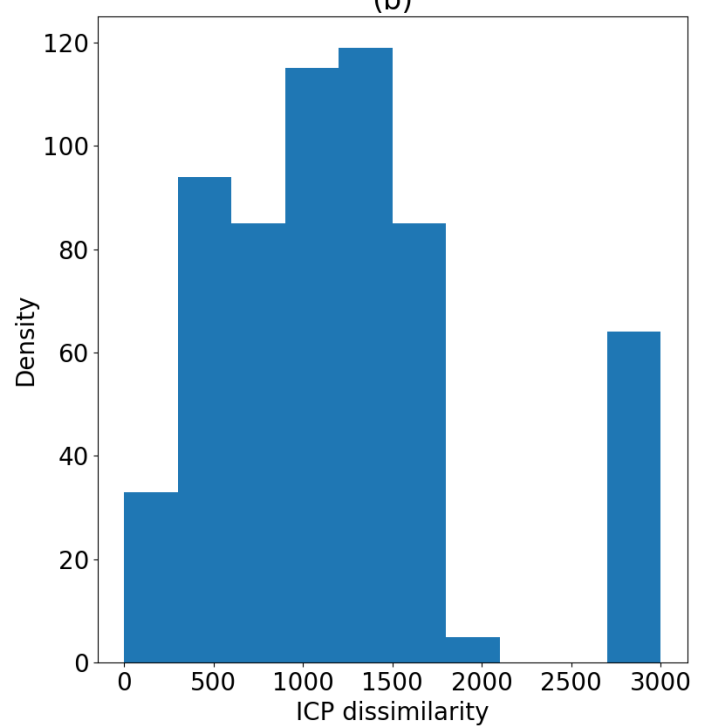

Figure 3: Histograms of dissimilarities to a short medoid. (a) corresponds to in the unclipped dissimilarities. For (b), dissimilarities were clipped to 3000 , deplacing the density peak initially around 20000 toward 3000 .

It might be observed, however, that always predicting an empty basket of product would lead to a perfect production score, while predicting a basket with a very high number of each product would lead to a perfect prediction score. These two scores need, therefore, to be balanced inside the predictionproduction score, naturally defined as:

$$
s^{\text {pre } \times \text { pro }}=s^{\text {pre }} \times s^{\text {pro }} .
$$

As for precision, recall and $F_{1}$, they are based on a redefinition of True positives (TP), False Positives (FP) and False Negatives (FN):

- TP is the number of lumbers which are present in both the real and predicted basket of a log, that is, $T P(y, \hat{y})=\sum_{i=1}^{p} \min \left(y_{i}, \hat{y}_{i}\right)$.

- FP is the number of lumbers present in the predicted basket of a log, but not in its real basket, that is, $F P(y, \hat{y})=\sum_{i=1}^{p} \max \left(\hat{y}_{i}-y_{i}, 0\right)$.

- FN is the number of lumbers present in the real basket of a log but not in its predicted basket, that is, $F P(y, \hat{y})=\sum_{i=1}^{p} \max \left(y_{i}-\hat{y}_{i}, 0\right)$.

precision, recall and F1 are then classically defined as:

$$
\begin{gathered}
\operatorname{precision}(y, \hat{y})=\frac{T P(y, \hat{y})}{T P(y, \hat{y})+F P(y, \hat{y})}, \\
\operatorname{recall}(y, \hat{y})=\frac{T P(y, \hat{y})}{T P(y, \hat{y})+F N(y, \hat{y})},
\end{gathered}
$$

and

$$
F_{1}(y, \hat{y})=2 \frac{\operatorname{precision}(y, \hat{y}) \operatorname{recall}(y, \hat{y})}{\operatorname{precision}(y, \hat{y})+\operatorname{recall}(y, \hat{y})} .
$$

While these scores are estimated individually for each $\log$, the quantity of interest is, naturally, their average over the evaluation dataset.

\section{DESCRIPTION OF THE PREDICTORS}

Several model architectures are tested and compared. These individual models are later used to build different predictors ensembles.

\subsection{INDIVIDUAL MODELS}

There exists two popular methods for handling multiple outputs prediction in such a regression framework. (Borchani et al., 2015) refers to them as problem transformation methods and algorithm adaptation method. Problem transformation methods change the multiple outputs problem into several single output problems. In our case, it corresponds to training one MLP for each type of lumber in the basket of products. On the contrary, algorithm adaptation methods use algorithms designed or modified to handle si- 
multaneously all the outputs. While problem transformation methods use simpler models, which might lead to better individual accuracy and smaller training time, they cannot be expected to handle dependencies among the outputs. Both of these strategies are tested in this paper.

\subsubsection{Multiple Input, Single Output (MISO)}

MISO models belong to the problem transformation family. Different MLPs are trained separately for each one of the 19 dimensions of the output vector. Therefore, 19 such individual MLPs, or MLP ensembles, are necessary to predict the whole vector. These MLPs are composed of one entry layer of size 21 . The number of neurons in the single hidden layer was fixed to 2 by trials and errors. To increase it even to 3 neurons leads to a strong overfitting of the learning base by the MLPs. The activation function of the hidden layer is always a hyperbolic tangent. However, several common activation functions for the output layer are compared: linear, sigmoid and hyperbolic tangent. An inconvenient of the linear output activation is that it allows the prediction of negatives lumber quantities, which have no reality in practice. Once trained, the prediction of these networks were therefore clipped to 0 when negative. Since the sigmoid and hyperbolic tangent have values between 0 and 1 , or between -1 and 1 respectively, the outputs where scaled accordingly for training with min-max transformations. These activation functions have the advantage that they constrain the inversely transformed prediction to stay between 0 and the maximum value encountered in the training set. A Relu activation function was similarly tested. However, it appears in practice that, in the MISO case, this activation leads to null gradients extremely early during the training process, sometimes even during the initial iteration. The sparsity of the output and the fact that the Relu is constant for negative values make it, indeed, possible to initialize the network at a point where the gradient is null. In particular, over 150 such models trained for the different outputs, $27 \%$ run less than 5 iterations of the optimization algorithm used before triggering a stopping condition. A discussion about the trainability of Relu-based NN, and the problem of dying neurons, can be found in (Shin and Karniadakis, 2020). MISO models with Relu activation are therefore not studied further in this paper.

\subsubsection{Multiple Input, Multiple Output (MIMO)}

Contrary to the MISO models, MIMO belong to the algorithm adaptation family, as all outputs are predicted simultaneously by the same MLP. Similarly to the MISO case, the input layer is of size 21. However, the number of neurons in the hidden layer was only reduced to 5 neurons. Similarly to what was tested in the MISO case, linear, sigmoid, hyperbolic tangent and Relu activation functions are tested for the output layer.

Table 1 summarizes the different architectures, and in particular provides the number of weights for each model. Since the limited size of the problem considered allows it, all neural networks were trained using Levenberg-Marquardt algorithm (Yu and Wilamowski, 2011), rather than a gradient descent. As explained for example by these authors, this algorithm is fast, as long as the matrix considered in its iteration are not too big, and it has stable convergence. Initialization of the networks was done using the NguyenWidrow algorithm (Nguyen and Widrow, 1990).

\subsection{ENSEMBLE SELECTION}

Creating ensembles is a commonly used method to improve on the results of a set of predictors (as in, to increase or minimize a chosen set of scores, for example the MSE in this paper). In particular, NN ensembles were introduced in (Hansen and Salamon, 1990). It is based on the fact that when training multiple NN with variations on the training method, for example different initializations, the training of these $\mathrm{NN}$ will converge toward different local minimums. Popular methods to introduce variety into the training of ML predictors include bagging (Breiman, 1996), boosting (Freund et al., 1996) or the Random Subspace method (Ho, 1998).

Additionally, numerous methods have been proposed to select predictors from a set of previously trained models. (Thomas et al., 2018) reports that two such families of methods either perform a selection based solely on a performance score, for example the MSE, or perform a selection based on both a performance scores and a diversity score. However, when comparing two such methods on their industrial dataset, they found no significant advantage to the use of a diversity based method, compared to the use of a performance based method.

In the following of this paper, we will therefore focus on a method to select MLPs for the ensembles based on the MSE score only. This method proceed as follow:

1. Evaluate the MSE of all the MLPs on a validation set and order them from best to worst.

2. from the best to the worst model, add them to the ensemble, and evaluate the ensemble MSE on the validation set.

3. Select the ensemble showing the lowest MSE. 


\begin{tabular}{|c|c|c|c|}
\hline Model & hidden layer size & number of weights & output activation \\
\hline \multirow{4}{*}{ MIMO } & \multirow{4}{*}{5} & \multirow{4}{*}{224} & Hyperbolic tangent \\
\hline & & & Sigmoid \\
\hline & & & Linear \\
\hline & & & Relu \\
\hline \multirow{3}{*}{ MISO } & \multirow{3}{*}{2} & \multirow{3}{*}{47} & Hyperbolic tangent \\
\hline & & & Sigmoid \\
\hline & & & Linear \\
\hline
\end{tabular}

Table 1: Summary table of the individual models being compared in this study

The prediction of an ensemble are here the averaged predictions of its individual elements. Additionally, 150 individual $\mathrm{NN}$ were trained with random initialization for each output of each of the MISO models, and 150 other for each of the MIMO models.

\section{RESULTS AND DISCUSSION}

This section presents the experimental results of our different models, first taken individually and later used to build ensembles. Considering that, in practice, a basket of products is a vector of integer, any potential negative predicted value was set to 0 , and outputs were rounded to the nearest integer. This additionally favor the emergence of null outputs which play an important role in the evaluation scores, especially the prediction, production, and prediction-production scores.

\subsection{Individual models}

150 models where trained for each architecture. Figure 4 shows the MSE of all the trained models, evaluated on the validation set. In the MISO case, predictions of a complete output vector where built by associating the predictions of the individual MISO in training order. The first MISO trained for all 19 outputs are used to generate a complete predictor, then the second MISO trained for all 19 outputs generate a second predictor, and so on. This figure shows, overall, an advantage of the Sigmoid activation function, both for MIMO and MISO models. Their MSE are, indeed, generally lower and present lower variation than other architectures.

Table 2 presents the scores of the best MIMO and MISO models with different outputs activation functions, and their asymptotic 95\% confidence intervals. These scores are evaluated on the evaluation set. These models were selected as being the ones with the lowest squared error over the validation set, among the 150 models with same architecture. In particular, in the MISO case, a complete prediction of the output vector is done by concatenation of the MISO which perform the best on their respective individual output. This table also displays, for each architecture, the total time needed to train all the 150 models. The models were implemented in Python and trained on an intel Core i7 vPRO $10^{\text {th }}$ generation $\mathrm{CPU}$ at $2.70 \mathrm{GHz}$. Matrix operations are performed with the library Numpy. It appears than on the dataset used in this study, the MISO model with sigmoid output performs best. In particular, it has the highest prediction-production score and $F_{1}$ and lowest MSE. It might be noticed that, similarly, the model with higher prediction-production score and $F_{1}$ among the MIMO models only is the one with sigmoid output. While the selected models with Tanh architecture perform relatively well on the evaluation set, however, the results from figure 4 do not encourage the use of this particular activation function.

Additionally, one should be careful that these differences in score, despite appearing important, are not always statistically significant. This is partly due to the high variability of the scores, and extensive tests on a larger dataset would be required to conclude on a difference. Here, in particular, when comparing the $F_{1}$ scores of the MISO sigmoid models with the scores of all the other selected models using Wilcoxon signed rank test, it cannot be said to be statistically different, at a 5\% level, from the MISO model with hyperbolic tangent output (p-value at 18.9\%) and MIMO model with sigmoid output (p-value at 9.2\%). However, both the MIMO and MISO models with linear or Relu activation perform relatively poorly compared with models of the same family.

It may be noticed that MISO models are, in average, faster to train than MIMO models as they contain less parameters. In particular, a MISO sigmoid model is, in average, more than 200 times faster to train than a MIMO sigmoid. Even when considering that 19 such networks have to be trained to perform a prediction of the full basket, this is an improvement. Interestingly, models with hyperbolic tangent output are relatively slow to train, as it appears in practice that they need more iterations of the levenberg-Marquardt algorithm before triggering a stopping condition. 


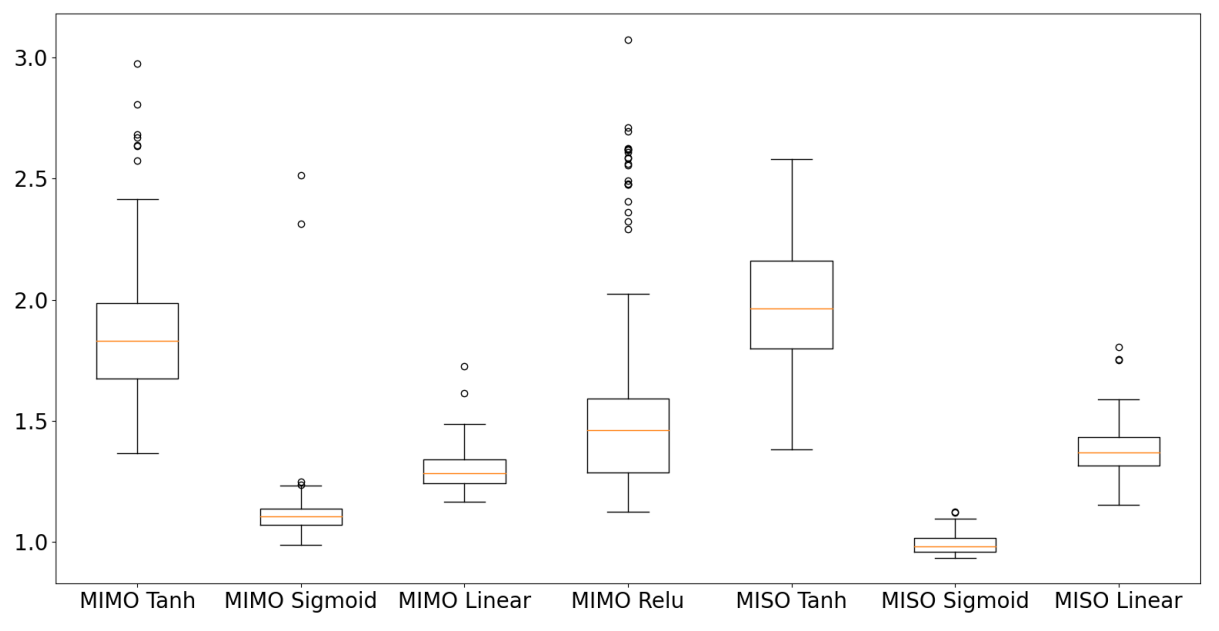

Figure 4: Boxplots of the MSE, estimated over the validation set, of the 150 models trained for each architecture. Prediction where inversely scaled for models having sigmoid or tanh output, to allow comparison.

\subsection{Ensemble}

Table 3 presents the averaged scores over the evaluation database of ensembles based on MLPs with different architectures. While the ensembles seem to improve upon the scores of several models, these differences are nearly never statistically significant, at a 5\% level, under Wilcoxon signed ranks test. In particular, the scores of the MISO sigmoid ensemble are never statistically different from the scores of the previously selected MISO sigmoid model, despite appearing even slightly lower than the scores of the individual models.

Concerning the size of the ensembles, the fact that the MISOs appears to need more models is due to the fact that 19 ensembles are needed to make a prediction of the complete output vector, and that all of that all of them are counted in table 3. As was observed in (Thomas et al., 2018), this method of building ensembles leads to parsimonious ensembles, containing only a small number of individual models. This is particularly the case of the models with sigmoid and linear outputs.

\section{CONCLUSION}

The set of features based on dissimilarities to medoids used to train MLPs in this study gives satisfactory results. This study furthermore compares several MLP architectures to the task of predicting baskets of products from 3D scans of wood logs. It appears that using an output activation function constraining the individual outputs in a certain value range leads to better evaluations scores, as the MLPs cannot predict unrealistic values. Therefore, both MIMO and MISO with sigmoid outputs work well and can be trained in reasonable time, with MISO performing seemingly better on our dataset. This is of particular interest when considering the fact that the sawmill configuration used in this paper processes a relatively small number of products. For example, (Morin et al., 2020) considers sawmills with as much as 83 products. The fact that ensembles barely increase the performances of the models is however surprising and may raise doubts on the possibility to consistently increase further the evaluation scores, at least when using only this set of medoid-based features.

Additionally, it appears that one should be extremely careful when working on this problem to avoid overfitting of the training database.

While in this paper, the features were selected as dissimilarities to class medoids, other logs could have been selected has references. To use metaheuristics like genetic algorithms or other feature selection methods during training time to select these logs might appears as a promising research direction for future works. Similarly, other classic ML algorithms, such as Random forest, will be investigated. 

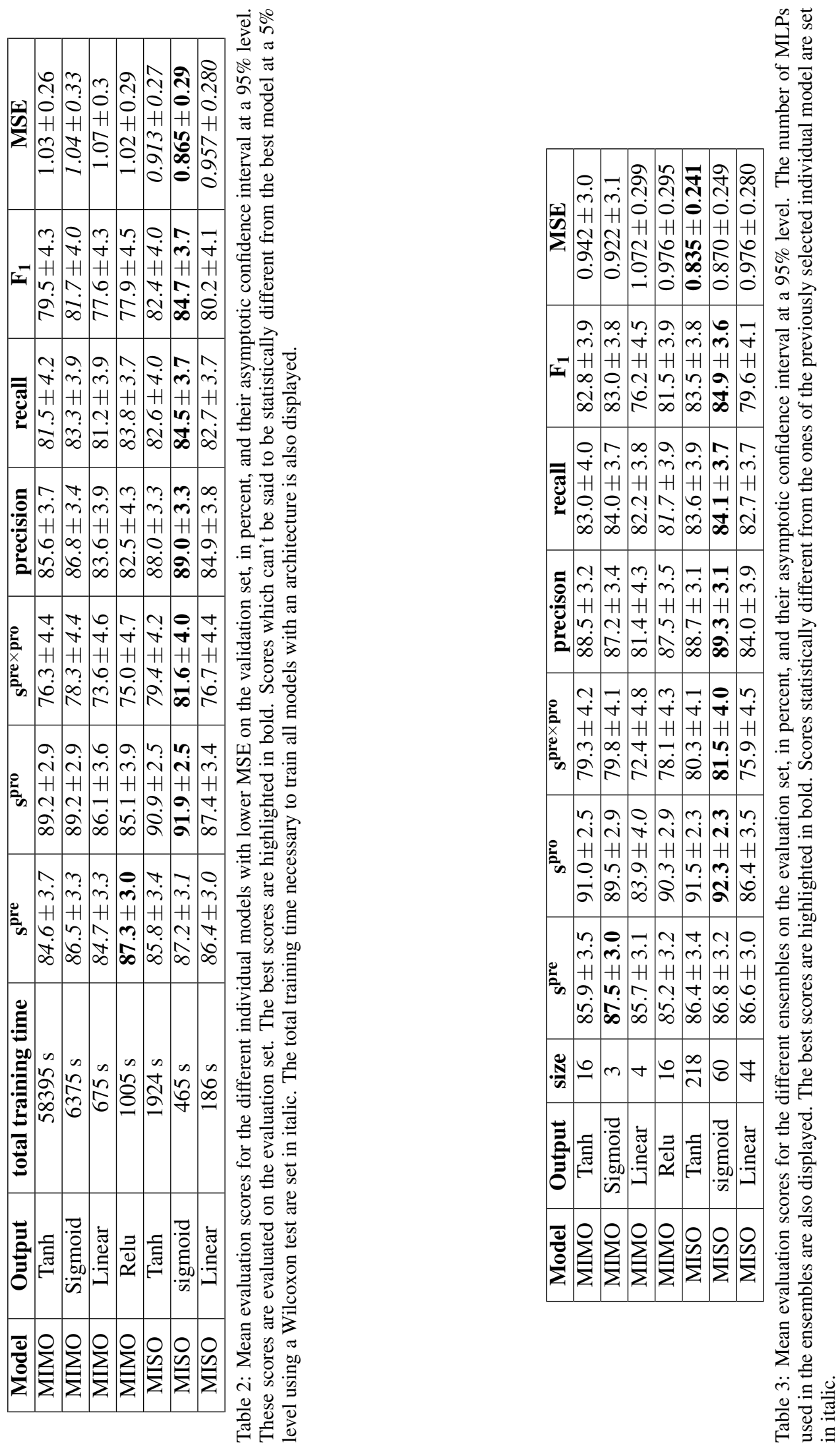


\section{ACKNOWLEDGEMENTS}

The authors gratefully acknowledge the financial support of the ANR-20-THIA-0010-01 Projet LOR-AI (lorraine intellgence artificielle) and région Grand EST. We are also extremely grateful to FPInnovation who gathered and processed the dataset we are working with.

\section{REFERENCES}

Besl, P. J. and McKay, N. D. (1992). A method for registration of 3-d shapes. IEEE Transactions on Pattern Analysis and Machine Intelligence, 14(2):239-256.

Borchani, H., Varando, G., Bielza, C., and Larranaga, P. (2015). A survey on multi-output regression. Wiley Interdisciplinary Reviews: Data Mining and Knowledge Discovery, 5(5):216-233.

Breiman, L. (1996). Bagging predictors. Machine learning, 24(2):123-140.

Cazzanti, L. (2009). Similarity Discriminant Analysis.

Chabanet, S., Thomas, P., El-Haouzi, H. B., Morin, M., and Gaudreault, J. (2021). A knn approach based on icp metrics for 3d scans matching: an application to the sawing process. In 17th IFAC Symposium on Information Control Problems in Manufacturing, INCOM 2021.

Del Frate, F. and Solimini, D. (2004). On neural network algorithms for retrieving forest biomass from sar data. IEEE Transactions on Geoscience and Remote Sensing, 42(1):24-34.

Duin, R. P. and Pekalska, E. (2009). The dissimilarity representation for pattern recognition: a tutorial. In Technical Report.

Freund, Y., Schapire, R. E., et al. (1996). Experiments with a new boosting algorithm. In $\mathrm{icml}$, volume 96, pages 148-156. Citeseer.

Goulet, P. (2006). Optitek: User's manual.

Hansen, L. K. and Salamon, P. (1990). Neural network ensembles. IEEE transactions on pattern analysis and machine intelligence, 12(10):993-1001.

Ho, T. K. (1998). The random subspace method for constructing decision forests. IEEE transactions on pattern analysis and machine intelligence, 20(8):832844.

Martineau, V., Morin, M., Gaudreault, J., Thomas, P., and Bril El-Haouzi, H. (2021). Neural network architectures and feature extraction for lumber production prediction. In The 34th Canadian Conference on Artificial Intelligence. Springer.

Morin, M., Gaudreault, J., Brotherton, E., Paradis, F., Rolland, A., Wery, J., and Laviolette, F. (2020). Machine learning-based models of sawmills for better wood allocation planning. International Journal of Production Economics, 222:107508.

Morin, M., Paradis, F., Rolland, A., Wery, J., Laviolette, F., and Laviolette, F. (2015). Machine learning-based metamodels for sawing simulation. In 2015 Winter Simulation Conference (WSC), pages 2160-2171. IEEE.

Morneau-Pereira, M., Arabi, M., Gaudreault, J., Nourelfath, M., and Ouhimmou, M. (2014). An optimization and simulation framework for integrated tactical planning of wood harvesting operations, wood allocation and lumber production. In MOSIM 2014, 10eme Conférence Francophone de Modélisation, Optimisation et Simulation.

Nguyen, D. and Widrow, B. (1990). Improving the learning speed of 2-layer neural networks by choosing initial values of the adaptive weights. In 1990 IJCNN International Joint Conference on Neural Networks, pages 21-26. IEEE.

Pekalska, E., Duin, R. P., and Paclík, P. (2006). Prototype selection for dissimilarity-based classifiers. Pattern Recognition, 39(2):189-208.

Qi, C. R., Su, H., Mo, K., and Guibas, L. J. (2017). Pointnet: Deep learning on point sets for $3 \mathrm{~d}$ classification and segmentation. In Proceedings of the IEEE conference on computer vision and pattern recognition, pages 652-660.

Schleif, F.-M. and Tino, P. (2015). Indefinite proximity learning: A review. Neural Computation, 27(10):2039-2096

Selma, C., El Haouzi, H. B., Thomas, P., Gaudreault, J., and Morin, M. (2018). An iterative closest point method for measuring the level of similarity of $3 \mathrm{~d}$ log scans in wood industry. In Service Orientation in Holonic and Multi-Agent Manufacturing, pages 433444. Springer.

Shin, Y. and Karniadakis, G. E. (2020). Trainability of relu networks and data-dependent initialization. Journal of Machine Learning for Modeling and Computing, 1(1).

Sylvain, J.-D., Drolet, G., and Brown, N. (2019). Mapping dead forest cover using a deep convolutional neural network and digital aerial photography. ISPRS Journal of Photogrammetry and Remote Sensing, 156:14 26.

Thomas, P., El Haouzi, H. B., Suhner, M.-C., Thomas, A., Zimmermann, E., and Noyel, M. (2018). Using a classifier ensemble for proactive quality monitoring and control: The impact of the choice of classifiers types, selection criterion, and fusion process. Computers in Industry, 99:193-204.

Thomas, P. and Thomas, A. (2011). Multilayer perceptron for simulation models reduction: Application to a sawmill workshop. Engineering Applications of Artificial Intelligence, 24(4):646-657.

Todoroki, C. et al. (1990). Autosaw system for sawing simulation. New Zealand Journal of Forestry Science, 20(3):332-348

Wenshu, L., Lijun, S., and Jinzhuo, W. (2015). Study on wood board defect detection based on artificial neural network. The Open Automation and Control Systems Journal, 7(1).

Wery, J., Gaudreault, J., Thomas, A., and Marier, P. (2018). Simulation-optimisation based framework for sales 
and operations planning taking into account new products opportunities in a co-production context. Computers in industry, 94:41-51.

$\mathrm{Yu}, \mathrm{H}$. and Wilamowski, B. M. (2011). Levenbergmarquardt training. Industrial electronics handbook, 5(12):1.

Zhang, B., Zhao, L., and Zhang, X. (2020). Threedimensional convolutional neural network model for tree species classification using airborne hyperspectral images. Remote Sensing of Environment, 247:111938. 